FORMATION Formation emploi

Revue française de sciences sociales

115 | Juillet-Septembre 2011

Le développement de l'aide à la personne : quelle professionnalisation?

\title{
Quels diplômes pour le secteur de l'aide à la personne?
}

What qualifications are available in the home care sector?

Welche Berufsabschlïsse im Sektor personenbezogener Dienst im Haushalt?

¿Qué diplomas para el sector de ayuda a las personas?

\section{Ghislaine Doniol-Shaw}

\section{(2) OpenEdition}

Journals

Édition électronique

URL : http://journals.openedition.org/formationemploi/3432

DOI : 10.4000/formationemploi.3432

ISSN : 2107-0946

Éditeur

La Documentation française

Édition imprimée

Date de publication : 23 septembre 2011

Pagination : 51-65

ISSN : 0759-6340

Référence électronique

Ghislaine Doniol-Shaw, «Quels diplômes pour le secteur de l'aide à la personne ? », Formation emploi [En ligne], 115 | Juillet-Septembre 2011, mis en ligne le 24 septembre 2011, consulté le 30 octobre 2020. URL : http://journals.openedition.org/formationemploi/3432 ; DOI : https://doi.org/10.4000/ formationemploi.3432

(c) Tous droits réservés 


\title{
Quels diplômes pour le secteur de l'aide à la personne?
}

\author{
Ghislaine Doniol-Shaw*
}

\section{Les besoins croissants des personnes fragilisées par l'âge ou les évènements de la vie impliquent de modifier l'organisation actuelle des formations afin qu'elles soient spécifiques à ces populations et qu'elles relèvent, simultanément, de l'aide et du soin.}

En France, les projections en besoins d'emplois à l'horizon 2015 ont mis en exergue les emplois « d'aide et de soins aux personnes fragiles » (Chardon et Estrade, 2007, p. 9), tout en soulignant les difficultés probables pour les pourvoir. Les emplois à domicile devraient connaître les tensions les plus vives. Ils correspondent en effet, à plus de $80 \%$, à de l'aide aux personnes âgées en perte d'autonomie. Ces tensions, confirmées en 2010 (Dares, 2010), sont à la fois dues aux besoins de nouveaux emplois pour faire face au vieillissement de la population et au besoin de renouvellement des emplois existants en raison du départ à la retraite de nombreuses salariées. Il s'agit en effet d'un métier où la moyenne d'âge est élevée du fait d'une entrée souvent tardive dans ce type d'emploi. 350000 postes devraient ainsi être à pourvoir entre 2007 et 2015 pour faire face aux besoins du grand âge, dont près de la moitié pour les seules aides à domicile. Ces besoins en emplois sont par ailleurs qualitativement en évolution ; l'accompagnement de personnes atteintes de maladies du type Alzheimer ou Parkinson ou présentant des handicaps importants (moteurs ou visuels en particulier) exigent en effet des connaissances spécifiques et des qualifications adaptées. Or, malgré ces besoins élevés en emplois d'aide auprès des personnes âgées et, plus généralement, auprès d'adultes fragilisés par l'âge ou les évènements de la vie (maladies, accidents), il n'existe aucune filière de formation spécifique. Il y a pourtant, comme nous le verrons, pléthore de diplômes dans le secteur de l'aide aux personnes. Toutefois, ils ont tous comme caractéristique d'être

* Ghislaine Doniol-Shaw est ergonome, ingénieur de recherche CNRS au LATTS (Laboratoire Techniques, Territoires et Sociétés), UMR (unité mixte de recherche) 8134, à l'université Paris-Est. Ses recherches interrogent les conséquences des changements techniques et organisationnels sur les conditions de travail et d'emploi et la santé, en prenant en compte le genre. Elle a récemment publié : Doniol-Shaw G. (2009), «L'engagement paradoxal des aides à domicile dans les situations repoussantes », Travailler, n²2, pp. 27-42 ; avec Le Douarin L. (2008), "L'accès des femmes aux emplois supérieurs de la fonction publique : une construction au croisement des itinéraires professionnels et familiaux », Politiques et management public, $n^{\circ} 26$, vol. 2, pp. 75-97. 


\section{Encadré 1}

\section{Terrains et méthodes}

Cet article repose sur deux recherches conduites sur le secteur de l'aide à domicile entre 2004 et 2008. La première a été réalisée en 2004-2005, dans le cadre d'un projet EQUAL, ayant bénéficié du financement du FSE (Fonds social européen). Elle a consisté à analyser le dispositif de VAE (validation des acquis de l'expérience) pour l'obtention du titre professionnel d'assistante de vie, délivré par le ministère du Travail par l'intermédiaire de l'AFPA (Association nationale pour la formation des adultes). Dans ce contexte, l'ensemble des diplômes et des formations de la filière professionnelle de l'aide à domicile et les métiers correspondants ont été questionnés.

Le dispositif a été analysé dans trois régions disposant d'au moins un centre AFPA doté d'une plate-forme de validation (formateurs et évaluateurs) ainsi qu'au sein d'associations employant des assistantes de vie. Outre l'étude des textes et travaux disponibles sur l'aide à domicile et sur la VAE, I'analyse s'est appuyée sur une série d'entretiens auprès de différents acteurs et sur l'observation de situations représentatives du processus de VAE. Des données quantitatives et qualitatives ont aussi été recueillies dans chaque région, afin d'évaluer l'efficacité du dispositif de VAE : taux de réussite, en lien avec les caractéristiques socioprofessionnelles des personnes concernées.

Des entretiens individuels ont été réalisés avec une vingtaine de personnes inscrites à différents niveaux dans le dispositif : en cours de formation, en cours de validation, ayant validé le titre.

Des entretiens ont également été conduits avec des formateurs et des responsables d'associations et avec une responsable ANPE (Agence nationale pour l'emploi, Pôle emploi à présent).

Différentes observations ont été effectuées:

- Participation à des réunions d'information sur la VAE Assistante de vie ;

- Participation à des réunions d'information sur le déroulement de la VAE et la démarche d'auto-positionnement, pour des candidats dont le dossier de VAE avait été accepté ;

- Observations du passage des différents Certificats de compétence professionnelle composant le titre d'Assistante de vie, dans un centre AFPA.

La seconde recherche a été réalisée en 2006-2007, en réponse à un appel d'offre du Service des droits des femmes et de l'égalité qui dépend du ministère du Travail, des Relations sociales et de la Solidarité. L'appel d'offre portait sur les " parcours promotionnels de femmes dans les services à la personne ". II s'agissait d'identifier des leviers qui permettraient, dans les emplois d'aide à domicile :

1. La mise en place de parcours de qualification professionnelle des femmes débouchant sur la promotion professionnelle ;

2. De limiter les temps partiels contraints.

La recherche s'est intéressée aux parcours d'emploi, de travail, de formation et de qualification des femmes salariées dans des structures associatives du secteur de l'aide à domicile. Six associations, appartenant à une même fédération, ont été enquêtées en distinguant région parisienne/province et zones rurales/zones urbaines.

Des entretiens biographiques enregistrés, d'une durée de trois heures environ, ont été réalisés auprès de 55 intervenantes à domicile expérimentées, c'està-dire ayant au moins cinq ans d'ancienneté dans le métier ou seulement trois ans pour les moins de 30 ans. L'emploi du temps hebdomadaire et ses caractéristiques précises (personnes aidées, tâches effectuées, relation avec les personnes...) ont été recueillis systématiquement.

Treize entretiens semi-directifs ont aussi été menés auprès d'acteurs associatifs (direction et encadrement intermédiaire principalement) et deux entretiens ont été réalisés avec des acteurs de l'Agence nationale des services à la personne - ANSP. 
orientés à la fois vers les besoins de la petite enfance et vers les besoins des personnes adultes fragilisées et/ou vieillissantes ; de même, nombre d'entre eux relèvent moins de l'aide que du service, orientant les emplois correspondants du côté du service domestique. Ajoutons que, hormis un diplôme de niveau IV (baccalauréat), tous les diplômes sont de niveau $\mathrm{V}$ ou $\mathrm{V}+$, c'est-à-dire correspondant à un CAP ou un BEP (respectivement certificat d'aptitude professionnelle et brevet d'études professionnelles).

Cet article se propose d'interroger le paradoxe entre un besoin de formation qualifiante, avéré et urgent, et l'absence de filière susceptible d'y répondre. Pour ce faire, nous mobiliserons des travaux conduits dans le secteur de l'aide à domicile. Ces travaux interrogent, d'une part, les conditions de la validation des acquis de l'expérience pour l'obtention du titre professionnel d'assistante de vie (Doniol-Shaw, 2004) ; d'autre part, les caractéristiques des parcours professionnels, de la formation et des situations de travail occupées par des salariées expérimentées d'associations d'aide à domicile (Doniol-Shaw, Lada, Dussuet, 2007) ( $c f$. encadré 1).

D'abord, nous définirons ce que nous entendons par « aide à la personne ", c'est-à-dire quelles sont les activités professionnelles réelles auxquelles cette expression correspond ; puis nous traiterons de l'organisation de ce secteur et des évolutions importantes qu'il a connues au cours des dix dernières années.

Nous présenterons ensuite l'ensemble des diplômes reconnus dans le secteur de l'aide à la personne et leur place dans les différentes conventions collectives concernées.

Nous discuterons alors des conséquences de la composition de ces diplômes et de leur prise en compte dans les conventions collectives relatives au secteur de l'aide à la personne sur la reconnaissance de la qualification et de l'expérience des salariées et sur leurs possibilités d'accès aux diplômes par la VAE (validation des acquis de l'expérience).

Enfin, nous examinerons les caractéristiques des parcours professionnels et de formation des salariées travaillant auprès de personnes fragiles. Nous nous appuierons sur ces parcours et sur les souhaits exprimés par ces salariées pour formuler des hypothèses quant aux formations qui seraient susceptibles de répondre aux exigences du travail d'aide auprès des personnes fragiles.

\section{L'AIDE À LA PERSONNE : DE QUOI PARLE-T-ON?}

Nous considérerons ici le terme d'aide à la personne dans son sens le plus précis, celui qui signifie aider quelqu'un à faire quelque chose. Dans la notion d'aide, il y a ainsi celle de participation conjointe à la réalisation d'une tâche. L'aide se définit en étroite relation avec les caractéristiques de la personne aidée, car l'aide doit s'y ajuster de façon permanente. Le travail d'aide, même s'il porte sur des activités définies, est ainsi constamment interrogé et repensé en fonction de l'état de la personne avec qui il s'effectue et non pas pour qui il s'effectue car, dans ce dernier cas, c'est de « service » et de « client » qu'il faut parler. C'est l'attention portée à la singularité de la personne, dans l'instant, qui est au cœur du travail d'aide (Pattaroni, 2005 ; Lada, 2010).

Quantitativement, l'évolution des besoins d'aide aux personnes est importante ; cependant, s'agissant des personnes destinataires de l'aide, c'est la continuité qui prévaut au cours des vingt dernières années. Il s'agit en effet avant tout et massivement d'aide à domicile, concernant à plus de $80 \%$ des personnes âgées ; environ $10 \%$ de l'aide à domicile est relative aux familles, à travers principalement la garde d'enfants au domicile des parents ${ }^{1}$, et $10 \%$ concerne les personnes adultes handicapées ou malades (Bressé, 2004). Les institutions accueillent en majorité des personnes dont le niveau de dépendance est élevé ; dès lors, le terme d'aide n'est guère approprié aux activités des personnels qui sont plus souvent dans la situation de faire « à la place » ou « pour » les personnes plutôt que de faire « avec » elles. En revanche, le terme d'aide reste approprié dans le cas des interventions auprès de résidents dans des foyers-logements. En effet, ces derniers, s'ils peuvent bénéficier de certains services collectifs, notamment de restauration, peuvent vivre en toute autonomie dans

\footnotetext{
${ }^{1}$ Seulement $1 \%$ des très jeunes enfants sont gardés au domicile des parents par une personne extérieure à la famille, rémunérée à cette fin. Note de veille $\mathrm{n}^{\circ} 107$ de juillet 2008 , du Centre d'analyse stratégique sur les modes de garde des enfants.
} 
leur appartement où ils disposent d'une cuisine et d'une salle de bains.

La prise en compte, dans les politiques sociales, de la croissance des besoins d'aide à domicile des personnes âgées s'est effectuée au cours des années 90. Ainsi, l'année 1997 voit la création de la prestation spécifique dépendance (PSD). Elle permet aux personnes âgées de financer des heures d'aide à domicile en lien avec le plan d'aide qui accompagne la délivrance de la prestation par le conseil général. La création de l'allocation personnalisée d'autonomie (APA) en 2002, en remplacement de la PSD, a accéléré la croissance des besoins d'aide à domicile, d'une part en élargissant la proportion des bénéficiaires (inclusion du GIR $4^{2}$ ) ; d'autre part, en ne soumettant pas l'aide au recours sur succession, c'est-à-dire en ne la récupérant pas sur l'héritage du bénéficiaire de l'aide après son décès, comme c'était le cas pour la PSD. Depuis 2003, on observe une augmentation constante et régulière des bénéficiaires de l'APA à domicile, de l'ordre de 3 à 3,5 \% par an. En 2009, ce sont ainsi près de 700000 personnes de plus de 60 ans (dont $85 \%$ de plus de 75 ans et $37 \%$ de plus de 85 ans), vivant à leur domicile, qui bénéficient de l'APA, et à travers elle du travail d'une aide à domicile (Debout et Lo, 2009).

La population principalement concernée par l'aide à domicile n'a pas changé ; cependant, les caractéristiques des personnes âgées demeurant à leur domicile ont, quant à elles, quelque peu évolué, modifiant la nature des besoins d'aide. En effet, la perte d'autonomie intervient plus tardivement; cependant, certaines pathologies du grand âge, liées à des déficiences cognitives ou physiques, deviennent plus lourdes à gérer. Avec le maintien à domicile de personnes plus dépendantes et aussi souvent plus isolées, en raison de l'éloignement géographique de la famille ou de la perte du conjoint, le travail des aides à domicile s'est ainsi éloigné d'une fonction d'aide-ménagère ; et ce au profit d'une fonction d'aide à la personne, dans le sens d'une aide à l'accomplissement des actes essentiels de la vie quotidienne (se laver, s'habiller, se nourrir, entretenir sa maison, se déplacer...) ; l'objectif visé étant la préservation, le plus longtemps et le mieux possible,

${ }^{2}$ GIR pour Groupe Iso Ressource, correspondant à une grille d'évaluation du degré de dépendance d'une personne allant de 1 (dépendance la plus forte) à 6. L'APA est délivrée aux personnes classées entre 1 et 4 . de l'autonomie des personnes, condition essentielle de leur maintien à domicile.

Cette évolution des besoins d'aide aux personnes âgées s'est accompagnée de certaines transformations conduites par l'État et les employeurs, notamment les associations.

\section{DES TRANSFORMATIONS EN LIEN AVEC L'ÉVOLUTION DES BESOINS D'AIDE}

Deux transformations importantes sont intervenues en 2002, c'est-à-dire simultanément à la mise en œuvre de l'APA : la création d'un nouveau diplôme et la signature d'un accord de branche. Des évolutions apparaissent également dans le cadre de l'emploi direct d'un salarié par un particulier.

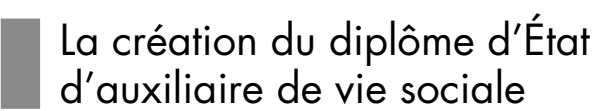

Le DEAVS, diplôme de niveau $\mathrm{V}+(\mathrm{CAP}+)$, délivré par le ministère des Affaires sociales sous couvert de la DASS (Direction des affaires sanitaires et sociales), est créé en mars 2002. Il remplace le CAFAD (Certificat d'aptitude aux fonctions d'aide à domicile), créé en 1988, mais qui n'était accessible qu'en formation continue. Dès sa création, le DEAVS est accessible par la formation initiale - à la condition d'avoir au moins 18 ans - ou par la formation continue et la VAE; cette dernière voie étant créée par la loi de modernisation sociale, datant elle aussi de 2002. La création du DEAVS valide l'exercice d'activités directement liées au corps, et en tout premier lieu l'aide à la toilette ; même si ces activités, bien que non autorisées, étaient, dans les faits, souvent réalisées auparavant par les intervenantes à domicile ${ }^{3}$. Cette évolution est déterminante pour l'exercice du métier et sa reconnaissance, comme en témoigne une salariée interviewée : «Le DEAVS a tout changé. (...) Lorsque la personne nous demandait de lui laver le dos ou de lui faire un bain de pieds, car

\footnotetext{
${ }^{3}$ Les salariés de l'aide à domicile étant à $98 \%$ des femmes, nous emploierons systématiquement le féminin pour les désigner (Dussuet, 2005).
} 
pour elle c'était impossible, on le faisait, mais nous n'avions pas le droit. C'est difficile de dire non à une grand-mère. Moi je me sentais capable de faire ça et je trouvais que cela faisait partie de mon métier. (...) Le métier a évolué, maintenant on peut faire la toilette tous les jours. Étape par étape et on y arrive. L'aide à la personne est primordiale, puis l'environnement. » (49 ans, CAFAD, 18 ans d'ancienneté)

Cependant, bien que le DEAVS ait été créé en lien direct avec l'APA, propre aux personnes âgées, il s'agit d'un diplôme polyvalent, c'est-à-dire qui couvre tout le spectre des personnes susceptibles d'être prises en charge à domicile, des tout petits aux personnes âgées.

\section{La signature d'un accord de branche de l'aide à domicile}

En mars 2002, certaines fédérations d'associations d'aide à domicile signent un accord de branche portant sur l'emploi et les rémunérations des salariés de l'aide à domicile. Dans cet accord, le DEAVS est classé au niveau $C$ dans la filière « intervention », qui comporte quatre niveaux, de $\mathrm{A}$ à $\mathrm{D}$. Il est au même niveau et suit strictement la même grille que le diplôme d'aidesoignante qui est inscrit dans la filière « soins ». Cet accord présente par ailleurs une particularité par rapport aux conventions collectives habituelles. Il lie, pour les emplois d'intervention, le classement dans la grille à la détention d'un diplôme reconnu par la branche. Cela signifie que seul le niveau A est accessible sans aucun diplôme, les niveaux B, C et D n'étant accessibles que si l'on est détenteur de l'un des diplômes admis en reconnaissance.

On est donc dans un régime conventionnel équivalent à celui des professions réglementées, comme pour les aides-soignantes, les infirmières ou les éducateurs, bien que l'emploi des salariées ne soit pas protégé par un statut. Dès lors, cet accord ne permet pas d'évoluer dans la grille de qualification en dehors de l'obtention d'un des diplômes reconnus. Par ailleurs, le changement devient automatique, dès lors que l'on obtient l'un de ces diplômes. Toutefois, le passage d'un niveau à l'autre se fait au premier échelon du niveau atteint ; cela signifie que l'ancienneté acquise dans les niveaux inférieurs n'est pas reconnue. Si celleci est importante, compte tenu du recouvrement des grilles de chaque niveau et d'une faible différence de progression entre échelons selon les niveaux, le gain, pour les salariées, peut-être très faible.

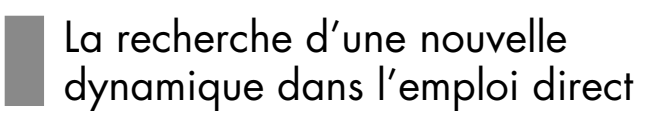

L'aide à domicile relève aussi de salariées travaillant dans le cadre de l'emploi direct, la personne aidée étant alors l'employeur. En 2008, 63 \% des salariées intervenant auprès de personnes âgées ou fragilisées sont directement employées par au moins une des personnes qu'elles aident, $24 \%$ étant exclusivement employées sous cette forme (Marquier, 2010). Dans ce cas, les salariées dépendent de la branche professionnelle des salariés du particulier employeur, qui a son propre système de classification et des diplômes qu'elle a fait homologuer. La FEPEM (Fédération $\mathrm{du}$ particulier employeur) cherche depuis quelques années, sous l'impulsion du plan Borloo de 2005 de développement des services à la personne, à revaloriser le statut des emplois à domicile, notamment en développant la formation. Sa perspective reste toutefois plus celle d'une valorisation d'un service (ainsi que nommé dans le plan Borloo) que d'une aide; cette situation est fortement renforcée par le lien de subordination qui existe entre la salariée et la personne aidée qui est aussi l'employeur.

La création de l'APA a donc accru la demande d'aide à la personne, quantitativement mais aussi qualitativement ; elle a en effet favorisé la possibilité de continuer à vivre à domicile malgré un niveau de dépendance important. Certaines mesures ont été prises pour répondre à ces nouveaux besoins qui ont conduit à une évolution du travail des aides à domicile et à des exigences nouvelles en matière de formation et de qualification. On constate cependant qu'il n'y a pas eu de véritable remise à plat de l'existant; notamment, le nouveau diplôme créé, le DEAVS, est venu s'ajouter aux diplômes existants et surtout les spécificités de

\footnotetext{
${ }^{4}$ Les salariées peuvent cumuler plusieurs statuts, c'est-à-dire travailler en tant que salariées d'une structure employeuse, le plus souvent associative, auprès d'une ou plusieurs personnes et être parallèlement en emploi direct, là aussi auprès d'une ou de plusieurs personnes, qui sont alors autant d'employeurs. L'enquête DREES indique que $24 \%$ des aides à domicile ne travaillent qu'en emploi direct, tandis que $39 \%$ travaillent à la fois en emploi direct et comme salariées d'une structure employeuse. $37 \%$ sont exclusivement salariées d'une structure, presque exclusivement associative.
} 
l'aide aux personnes âgées ou fragilisées n'ont pas été considérées comme devant faire l'objet d'une formation spécialisée. Le DEAVS est en effet un diplôme polyvalent, intégrant les besoins de prise en charge des tout-petits comme des personnes âgées ; pourtant, moins de $10 \%$ des activités à domicile concernent la garde des jeunes enfants et, dans ce cas, les salariées, lorsqu'elles sont diplômées, disposent plus souvent du CAP Petite enfance, diplôme spécifique à cette activité.

Pour éclairer ces propos, nous présentons et discutons les diplômes reconnus dans les deux cadres d'exercice d'aide à la personne à domicile : celui de la branche professionnelle de l'aide à domicile (cadre associatif) et celui de la branche professionnelle des salariés du particulier employeur.

\section{UNE MULTIPLICITÉ DE DIPLÔMES DE PREMIER NIVEAU}

Le panorama des formations et des diplômes reconnus par le secteur de l'aide à la personne à domicile est vaste et complexe. En effet, il n'est nullement harmonisé et les modes de reconnaissance diffèrent selon la qualité de l'employeur - employeur particulier, association, entreprise. En même temps, il est remarquable d'observer qu'à l'exception d'un seul diplôme de niveau IV, tous les diplômes sont de niveau V, sans bénéficier néanmoins de la même reconnaissance dans les grilles de classification.

\section{La reconnaissance des diplômes dans les grilles de classification}

Quatre ministères dispensent des diplômes reconnus, à différents niveaux, dans la grille de classification de la branche professionnelle de l'aide à domicile, couvrant le champ associatif.

La branche professionnelle des salariés du particulier employeur a de son côté créé, en février 1998, le titre d'employé familial polyvalent. Ce titre professionnel de niveau $\mathrm{V}$ a été inscrit au Répertoire national des certifications professionnelles (RNCP) en mars 2005, en même temps que deux Certificats de qualification professionnelle (CQP) : un CQP Garde d'enfants à domicile et un CQP Assistante de vie, centré sur les personnes âgées et rebaptisé, en 2009, « Assistant de vie dépendance ». Ces CQP constituent une spécialisation par rapport au titre d'employé familial polyvalent. Ils conduisent à un classement au niveau supérieur dans la grille de classification en 5 niveaux du particulier employeur (niveau 3 contre niveau 2). Dans cette grille, aucun des diplômes d'État n'apparaît. Inversement, la branche professionnelle de l'aide à domicile reconnaît au niveau $\mathrm{B}$ le titre d'employé familial polyvalent.

Il n'existe pas, à l'heure actuelle, de convention collective couvrant les salariés d'une entreprise privée de services à la personne. La négociation est en cours depuis 2007.

Ainsi, dans le cadre des emplois couverts par l'accord de branche de l'aide à domicile, un seul diplôme de niveau IV permet le classement en $\mathrm{D}$, celui de Technicienne de l'intervention familiale et sociale, délivré par la DASS (Direction des affaires sanitaires et sociales). Les emplois correspondants sont, pour la plupart, exercés auprès des familles en difficulté, leur employeur étant alors le plus souvent le Centre communal d'action sociale. Leur présence dans les associations d'aide à domicile est de fait en constante diminution (Dussuet, 2005).

Parmi les diplômes de niveau V, seuls deux d'entre eux donnent accès au niveau $\mathrm{C}$ de la classification de l'aide à domicile, tous les autres ne conduisant qu'au niveau B. Il s'agit du BEP Carrières sanitaires et sociales associé à la mention complémentaire Aide à domicile, datant de 1995, et du DEAVS datant de 2002. Les salariées détentrices du CAFAD sont classées en C par équivalence de leur diplôme avec le DEAVS.

En outre, aucun des diplômes délivrés par le ministère de l'Agriculture ne bénéficie d'un classement en C ; pourtant, historiquement, il est le premier à avoir en quelque sorte professionnaliser les emplois familiaux (Dussuet, 2005).

Ainsi, une même activité est couverte par plusieurs diplômes, titres ou certificats de niveau $\mathrm{V}$, avec néanmoins une hiérarchie entre ces diplômes et, en correspondance, dans le classement des salariés au sein de la branche professionnelle de l'aide à domicile. Quant à la branche professionnelle des salariées du particulier employeur, elle classe les salariées uniquement en fonction de ses propres 
Tableau 1

Panorama des diplômes et leur reconnaissance dans la grille de classification de la branche professionnelle de l'aide à domicile

\begin{tabular}{|c|c|c|}
\hline & Diplômes délivrés et date de leur création & $\begin{array}{c}\text { Classement dans la grille } \\
\text { de classification } \\
\text { de la branche professionnelle } \\
\text { de l'aide à domicile }\end{array}$ \\
\hline Éducation nationale & $\begin{array}{l}\text { - BEP Carrières sanitaires et sociales - CSS (1993). } \\
\text { - BEP CSS + mention complémentaire Aide à domicile } \\
\text { MCAD (1995). } \\
\text { - CAP Petite enfance (1991). } \\
\text { - CAP Employé technique de collectivités (1975). }\end{array}$ & $\begin{array}{l}\text { B } \\
\text { C } \\
B \\
B\end{array}$ \\
\hline $\begin{array}{l}\text { Affaires sociales (DASS } \\
\text { - Direction des affaires } \\
\text { sanitaires et sociales) }\end{array}$ & $\begin{array}{l}\text { - Diplôme d'État d'auxiliaire de vie sociale - DEAVS } \\
\text { (2002). } \\
\text { - Diplôme d'État d'aide médico-psychologique - } \\
\text { DEAMP (1972). } \\
\text { - Diplôme d'État d'aide-soignante - DEAS (1956). } \\
\text { - Diplôme d'État de technicienne de l'intervention } \\
\text { sociale et familiale - DETISF (1974). }\end{array}$ & $\begin{array}{c}\text { C } \\
\text { B } \\
\text { B (en filière Intervention mais C } \\
\text { en filière Soins). } \\
\text { D }\end{array}$ \\
\hline $\begin{array}{l}\text { Affaires sociales (DTEFP } \\
\text { - Direction du travail, de } \\
\text { l'emploi et de la formation } \\
\text { professionnelle) }\end{array}$ & - Titre Assistante de vie aux familles (2000). & B \\
\hline Agriculture & $\begin{array}{l}\text { - BEP Services, spécialité services aux personnes } \\
\text { (1992). } \\
\text { - BEP Économie familiale et rurale (1972). } \\
\text { - CAP Services en milieu rural (2000). } \\
\text { - CAP Employé d'entreprise agricole, option employé } \\
\text { familial (1971). }\end{array}$ & $\begin{array}{l}\text { B } \\
B \\
B \\
B\end{array}$ \\
\hline
\end{tabular}

Source : Accord de branche de l'aide à domicile du 29 mars 2002 relatif aux emplois et aux rémunérations. Sigles : CAP : certificat d'aptitude professionnelle ; BEP : brevet d'études professionnelles.

diplômes, dont aucun ne dépasse le niveau V. Enfin, l'État introduit la concurrence entre ses ministères ou même au sein d'un même ministère (diplômes délivrés par la DASS ou la DTEFP). Il crée en effet des diplômes de même niveau et qui sont orientés vers les mêmes emplois, sans que ces diplômes bénéficient de la même reconnaissance dans les grilles de classification. De plus, dans le cadre de la branche professionnelle de l'aide à domicile, l'accès au DEAVS et au niveau $C$ n'est pas ouvert dans les mêmes conditions aux salariées classées en B et donc titulaires d'un diplôme correspondant. Selon le diplôme dont elles disposent, les équivalences obtenues sont en effet très différentes.

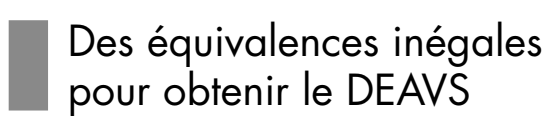

Le DEAVS est constitué de six modules correspondant à six domaines de formation. Selon les diplômes détenus, la dispense d'un ou de plusieurs de ces modules peut être obtenue.

Le diplôme d'État d'aide médico-psychologique (DEAMP) dispense de 4 modules. Le titre professionnel d'assistante de vie aux familles (AdV) ne dispense que de 2 modules alors que les compétences des deux métiers se définissent à l'identique 5 . Cela

5 On trouve ainsi sur le site de l'association ADAI 13:
(www.adai13.asso.fr/fiches/pro/pro_aide_a_domicile.htm) les
informations suivantes pour la définition des compétences : 
interroge sur les fondements d'une telle hiérarchie entre les deux diplômes. Ce sont les mêmes dispenses qui sont accordées aux titulaires d'un BEP Carrières sanitaires et sociales, d'un diplôme d'État d'aidesoignante ou du diplôme d'État d'assistant familial ${ }^{6}$.

Tous les autres diplômes permettant le classement en $B$ ne dispensent que d'un seul module.

À la diversité des diplômes et des équivalences entre eux, la branche professionnelle de l'aide à domicile ajoute la particularité de lier étroitement le classement dans la grille des emplois à la détention d'un de ces diplômes. Ce lien a de nombreuses conséquences sur l'embauche et la carrière des salariées.

\section{LES PARADOXES DU LIEN ENTRE DIPLÔMES ET CLASSIFICATION DANS LA GRILLE DES EMPLOIS}

L'accord de branche de l'aide à domicile lie donc le classement à la détention d'un diplôme. Cette valorisation des emplois passe exclusivement par la valorisation de certains diplômes; dès lors, elle néglige les salariées disposant d'une expérience professionnelle non validée par un diplôme reconnu par la branche. La voie de la VAE est certes ouverte aux salariées disposant d'au moins trois ans d'expérience ; néanmoins, l'obtention d'un diplôme par cette voie se heurte à deux types d'obstacles.

En effet, la VAE reste problématique pour les salariées qui disposent d'un faible capital scolaire initial et redoutent, de ce fait, «l'épreuve » de la VAE ou de toute forme de formation académique sanctionnée par un « examen » (Presse, 2004). Elle l'est d'autant plus lorsque les salariées ne bénéficient pas d'un accompagnement propre à leur donner confiance dans leur expérience et leurs capacités (Bonaïti, 2008) ; cette situation est de plus en plus fréquente, en raison des restrictions budgétaires au sein des associations.

«L'auxiliaire de vie : il lui sera demandé les mêmes compétences que celles demandées aux assistantes de vie».

${ }^{6}$ Ce diplôme, délivré par la DASS, a été créé en 2006. Il est destiné à des personnes désignées comme assistants familiaux, accueillant à leur domicile des mineurs ou de jeunes majeurs dans le cadre de l'aide sociale à l'enfance ou d'un placement judiciaire.
Le second obstacle tient au caractère polyvalent des diplômes, orientés à la fois vers les tout-petits et vers les adultes fragilisés. L'aide à domicile ne concernant pratiquement que de l'aide à des adultes fragilisés, essentiellement des personnes âgées, l'expérience professionnelle auprès des nourrissons est exceptionnelle. Autrement dit, l'expérience ne suffit pas à elle seule pour valider un diplôme. Dans les faits, la situation diffère quelque peu entre les deux diplômes réellement ouverts à la VAE : le DEAVS et le titre professionnel d'assistante de vie, AdV. En effet, dans la plupart des cas, les jurys du DEAVS, faisant en quelque sorte preuve de pragmatisme, acceptent que les salariées ne soient évaluées que sur leur activité auprès des adultes fragilisés et/ou âgés. Ceci est également rendu possible par le fait qu'aucun des 6 modules du DEAVS n'est spécialisé sur les seuls enfants.

Au contraire, la composition du titre AdV ainsi que les conditions de sa validation par la VAE imposent de faire la preuve de ses capacités à s'occuper autant de très jeunes enfants que d'adultes fragilisés. Le titre d'assistante de vie comporte en effet trois certificats de compétence professionnelle (CCP) distincts, à valider séparément :

1 - Assister les personnes dans la réalisation des actes de leur vie quotidienne ;

2 - Assurer la garde active des enfants et des bébés à (leur) domicile ;

3 - Assister les personnes dans la réalisation de leurs tâches domestiques.

Contrairement au DEAVS, où il s'agit pour les candidates de constituer un dossier professionnel qu'elles défendent ensuite devant un jury, la VAE AdV se présente sous la forme d'une mise en situation, dans le cadre reconstitué d'un appartement, pour chacun des $\mathrm{CCP}^{7}$. Un entretien devant un jury final intervient au terme de la validation de tous les CCP.

\footnotetext{
${ }^{7}$ Pour la validation des CCP 1 et 3 , la candidate doit suivre un scénario décrivant la situation de travail. Pour le CCP 1, la personne âgée ou dépendante est jouée par un comédien qui s'inscrit dans le scénario présenté au candidat. Pour le CCP 2, c'est un poupon qui représente le bébé et un membre du jury ou une personne extérieure joue, au début, le rôle de la mère, précisant certains aspects de la situation, questionnant la personne et, le cas échéant, répondant à ses questions.
} 
Ces modalités de validation de « l'expérience », qui font appel à la simulation (comédien, poupon) et qui proposent des scénarios peu crédibles (Doniol-Shaw, 2005-b), interrogent. En outre, on constate que lorsque les candidates ne disposent que d'une expérience professionnelle auprès des personnes âgées ou fragilisées, ce qui est presque toujours le cas, la majorité des échecs à la validation du titre $\mathrm{AdV}$ résulte de l'échec à la validation du CCP 2 relatif à la garde des enfants (Doniol-Shaw, 2005-a). Mais la seule validation des CCP 1 et 3 , représentative des capacités des salariées à exercer leur métier auprès des personnes âgées et/ ou fragilisées, ne permet pas le passage au niveau B, que seul le diplôme complet autorise. Cette situation est donc profondément paradoxale. Elle oblige en effet les salariées à faire la preuve de capacités dans un domaine dont elles n'ont pas l'expérience et vers lequel elles ne veulent pas s'orienter ; et ce d'autant plus qu'elles en connaissent les faibles débouchés et la concurrence avec des salariées disposant d'une formation spécifique à la petite enfance.

Quant au DEAVS, si les obstacles à son obtention par la VAE ne sont pas du même ordre, ils restent cependant importants. Ainsi, seulement $45 \%$ des salariées en emploi obtiennent ce diplôme lors du premier passage devant le jury. Pour les autres, il peut s'agir d'une validation nulle, dans $6 \%$ des cas, ou le plus souvent partielle, qui nécessite donc de repasser, parfois plusieurs fois, devant un jury pour compléter la validation. Ici, le risque d'abandon de la démarche s'accroît en cas d'échec répété (Marquier, 2008). Les jurys peuvent facilement mettre les candidates en difficulté. Ils sont ainsi susceptibles de les interroger sur des points sans rapport avec l'expérience rapportée dans le dossier ou encore, comme c'est souvent le cas, en posant des questions orientées vers des aspects médicaux, les présidences des jurys ou les représentants des formateurs étant souvent des infirmières. Le témoignage d'une directrice d'association d'aide à domicile illustre ces problèmes : « $L a$ deuxième année, on a eu un problème de jury, avec un jury fortement médical. Du coup, on a eu beaucoup d'échecs et ça a jeté une douche froide... L'année dernière (2006), on a senti encore que les jurys ne correspondaient pas vraiment à ce qui se passait. Il y avait des questionnements qui n'étaient pas vraiment dans le cœur de métier... »
Le lien entre le diplôme et la classification interdit aussi la reconnaissance, à l'entrée dans le secteur, d'une expérience dans l'aide aux personnes hors du secteur de l'aide à domicile, que ce soit en maison de retraite, à l'hôpital ou dans toute autre structure collective. La encore, le paradoxe est particulièrement fort. En effet, comme nous l'avons constaté dans nos enquêtes, cette expérience est à l'origine de leur recrutement dans l'aide à domicile.

Une autre particularité tient à la sélectivité des diplômes reconnus pour le classement des salariées. Ainsi, des salariées disposant d'un diplôme de niveau supérieur au niveau V, comme un bac Sciences et technologies de la santé et du social (ST2S) ou une maîtrise de psychologie, avec une spécialisation sur les personnes handicapées, se verront classées en A, comme les salariées sans aucun diplôme. Elles devront passer un diplôme de niveau inférieur à celui qu'elles détiennent pour progresser dans la grille... De plus, dans le cas de la possession d'un diplôme supérieur, il n'existe aucune équivalence avec les diplômes de la branche.

Ainsi, en se calquant sur le modèle des professions réglementées pour les emplois d'intervention situés dans les niveaux les plus bas de la grille ${ }^{8}$, l'accord de branche sur les classifications et les rémunérations de l'aide à domicile ne permet pas aux salariées de faire valoir une expérience non validée par un diplôme reconnu par la branche. De même, l'absence de formation qualifiante, exclusivement orientée vers les personnes âgées ou fragilisées, freine la possibilité d'obtenir un diplôme par la voie de la VAE et limite donc la promotion des salariées. La partie suivante approfondit la réflexion, d'une part, sur la composition des diplômes et leur inadéquation aux emplois proposés ; d'autre part, sur les souhaits et orientations des salariées.

\section{DES DIPLÔMES PROFESSIONNELS... POUR DES MÉTIERS QUI N'EXISTENT PAS}

L'analyse du contenu des diplômes professionnels reconnus pour l'accès aux emplois d'aide aux ${ }^{8}$ Ce ne sont en effet que ces emplois dont le classement est lié à
la détention d'un diplôme spécifique ; les emplois d'encadrement,
classés dans les niveaux supérieurs, ne suivant pas cette règle. 
personnes révèle qu'ils forment pour un métier qui n'existe pas, ou sinon à la marge. Il est en effet exceptionnel que des salariées exercent simultanément une activité auprès de jeunes enfants et de personnes âgées.

Dans la recherche que nous avons réalisée (DoniolShaw, Lada, Dussuet, 2007), environ le quart des salariées interviewées (55 au total ayant au moins 5 ans d'ancienneté) possédait l'expérience d'interventions auprès de différents publics, la majorité n'exerçant donc qu'auprès de personnes âgées. Dans cette diversité, notons que tous les publics étaient représentés, à l'exception des tout-petits (moins de 3 ans). Ce point est important car les contenus des formations les plus spécifiques de l'aide à domicile concernent, pour la partie consacrée aux enfants, essentiellement la prise en charge des bébés.

Ajoutons que la polyvalence à la fois envers les personnes âgées et les nourrissons ne correspond pas non plus aux souhaits des salariées. Dans notre enquête, la plupart des salariées interviewées ne désiraient pas travailler auprès de très jeunes enfants, estimant le plus souvent que cela devenait plus difficile avec l'âge : "Les enfants, je n'ai pas envie. C'est bon pour les plus jeunes. Moi, je ne les supporte pas. » (54 ans) ou encore : "Je pense qu'en ce qui me concerne, les enfants en bas âge, j'ai fait mon temps, place aux autres. » (38 ans). Même si certaines avaient pu émettre ce souhait, elles s'étaient trouvées confrontées à la réalité du marché qui les avait de facto orientées vers les personnes âgées. Elles étaient favorables à une diversification des publics mais leur demande visait principalement l'aide à des publics adultes atteints de maladie ou de handicap, situations que plusieurs d'entre elles avaient expérimentées et qu'elles souhaitaient voir se développer.

Enfin, l'examen des résultats de l'enquête de 2008 de la DREES sur les intervenantes au domicile des personnes fragilisées (Marquier, 2010), révèle que, parmi les $38 \%$ de salariées qui possèdent un diplôme du secteur sanitaire et social, $21 \%$ détiennent le DEAVS (ou le Cafad) et $8 \%$ le BEP Carrières sanitaires et social, ce qui représente $75 \%$ des diplômes détenus. Ce ratio est encore plus élevé pour les intervenantes salariées d'associations, dont $52 \%$ détiennent un diplôme du secteur sanitaire et social, puisqu'il est de $90 \%$ (68\% pour le DEAVS ou le Cafad et $22 \%$ pour le BEP CSS $)^{9}$. Le titre Assistante de vie est détenu par $8 \%$ des salariées diplômées et le BEP agricole Services aux personnes par moins de $4 \%$. Quant à la mention complémentaire Aide à domicile, qui conduit pourtant au classement en $\mathrm{C}$ dans l'accord de branche de l'aide à domicile, elle n'est détenue que par 1,3\% des diplômées.

Outre le constat de la forte proportion de salariées exerçant le métier sans détenir de diplôme reconnu par le secteur (62\% des salariées), on remarquera aussi que la diversité des formations reconnues pour l'accès à ces emplois ne recoupe pas les formations réellement détenues, essentiellement polarisées sur deux diplômes.

La primauté accordée à ces formations n'est pas sans lien avec leur contenu orienté vers l'aide et le «prendre soin », la plupart des autres formations reconnues relevant plutôt des services. Les « choix » de formation des salariées peuvent ainsi être lus comme le reflet de leurs choix d'orientation professionnelle vers le travail de «care », au sens du «prendre soin » et $\mathrm{du}$ « souci de l'autre » (Laugier, Molinier, Paperman, 2009) ; posture qui est plus ou moins en opposition avec les emplois de service qui peinent à échapper, surtout lorsqu'il s'agit d'emploi à domicile, à la dimension de domesticité, voire de servilité (Vasselin, 2002, Fraisse, 2009, Doniol-Shaw, 2009). Nous allons approfondir ces aspects, dans la partie suivante, et proposerons des orientations directement issues de la réalité observée sur le terrain.

\section{POUR UNE FORMATION QUI CONJUGUE L'AIDE ET LE SOIN}

Les diplômes majoritairement détenus par les salariées de l'aide à domicile se distinguent donc par leur orientation ; celle-ci s'inscrit dans le champ du sanitaire et du social et est de ce fait corrélée avec les soins à la personne, y compris les soins du corps. Le DEAVS a été créé spécifiquement pour permettre

\footnotetext{
${ }^{9}$ Notre enquête présentait des données semblables, $56 \%$ des salariées étant détentrices d'un diplôme du secteur (DEAVS ou Cafad dans $70 \%$ des cas).
} 
aux aides à domicile de réaliser des soins au corps et en tout premier lieu les aides à la toilette ; cela peut aller, comme nous l'avons souvent rencontré, jusqu'à faire des toilettes au lit. Le BEP Carrières sanitaires et sociales est, de son côté, le diplôme le plus souvent choisi par les élèves qui souhaitent ensuite présenter le concours d'aide-soignant, pour lequel ils sont dispensés des épreuves écrites d'admissibilité. Les autres diplômes reconnus par l'accord de branche de l'aide à domicile, comme le CAP employé de collectivité et les diplômes délivrés par le ministère de l'Agriculture - sauf le BEPA Services aux personnes qui est proche, dans son contenu, du BEP CSS, avec toutefois nettement moins de formation aux techniques du corps - relèvent donc davantage des activités de service dans lesquelles les dimensions matérielles (entretien des locaux, préparation et service des repas, accueil, vente...) priment sur les activités relationnelles et de soin.

Cette spécialisation sur les diplômes les plus orientés vers les activités du care indique en creux le type de connaissances nécessaires à la maîtrise des activités d'aide aux personnes. Le travail d'aide suppose en effet un constant travail d'évaluation de l'état physique et psychologique de la personne aidée, pour ajuster précisément l'aide à la situation de la personne, sur le moment. Or, des connaissances précises sont nécessaires à cette évaluation, que ce soit en matière d'hygiène, d'alimentation, de soins quotidiens, de psychologie, de risques spécifiques à ces populations fragilisées ou de pathologies rencontrées. Le diagnostic, outillé par ces connaissances, oriente les actions à réaliser, centrées sur la préservation de l'autonomie, le bien-être et la dignité de la personne.

Les formations susceptibles de soutenir de telles orientations ne sont pas déterminées par le lieu d'exercice du travail, le domicile ou l'institution. En effet, ce qui est en jeu c'est l'accompagnement de la vie et des activités quotidiennes qui la sous-tendent; et ce, dans une phase déclinante sans doute, mais qui n'entame pas inévitablement le désir de vivre, qui est aussi celui d'agir, quel que soit le lieu de vie. C'est en tout cas ce dont témoignent toutes les salariées que nous avons interviewées (Doniol-Shaw, Lada, 2009), même si nous n'en citons qu'une : "La dame qui a la maladie d'Alzheimer, hier, elle a fait du repassage avec moi, si vous saviez comme elle était contente.
C'est ça mon travail, préserver ce qui reste. » (48 ans, DEAVS, 3 ans d'ancienneté)

Ce travail d'aide a ainsi bien peu à voir avec ce qu'exigent les soins aux jeunes enfants et le souci de leur développement, qui visent non la préservation mais la conquête de l'autonomie. Ces besoins propres aux enfants sont d'ailleurs largement reconnus puisque des formations spécifiques y sont consacrées (CAP petite enfance, auxiliaire de puériculture, éducatrice de jeunes enfants, auxiliaire de vie scolaire...).

C'est donc vers la création de formations spécialement pensées pour répondre aux situations des adultes fragilisés, notamment par l'âge, qu'il nous semble nécessaire de s'orienter. Ces formations questionnent inévitablement la frontière actuellement tracée entre le sanitaire et le social et que l'on peut lire dans la séparation entre les tâches d'aide-soignante et les tâches d'auxiliaire de vie sociale. Les demandes de formation exprimées par les salariées visent majoritairement une meilleure connaissance des pathologies $\mathrm{du}$ vieillissement et des problèmes psychologiques qui les accompagnent. Elles concernent aussi souvent l'accompagnement des fins de vie dans le cadre du vieillissement comme dans celui des maladies incurables. Faute de se voir proposer de telles formations, car la formation continue leur est dispensée au compte-goutte, c'est bien souvent par elles-mêmes que les salariées trouvent les ressources pour acquérir les connaissances nécessaires. Elles évoquent ainsi l'usage intensif d'internet ou le suivi des émissions médicales à la télévision et à la radio ou encore la lecture des articles traitant de ces questions dans les journaux. Nous avons par ailleurs constaté que les salariées qui formulaient le projet de se former comme aide-soignante le faisaient moins pour s'orienter vers ce métier que pour consolider leurs connaissances et pouvoir durablement accompagner les personnes, en les suivant dans l'évolution de leur état de santé, comme l'évoque clairement l'une d'entre elles : "Pour moi, ce serait éviter de refuser un dossier parce qu'il n'y a pas les compétences, pas le diplôme. Ce serait donc une complémentarité vis-à-vis de mon métier. » (45 ans, Cafad, 15 ans d'ancienneté)

Sur la base de ces constats, nous avons formulé l'hypothèse d'une recombinaison possible des métiers d'aide-soignante et d'auxiliaire de vie. Il s'agit d'aller 
vers une « auxiliaire de vie soignante », dont les compétences permettraient un accompagnement au plus près des besoins des personnes, en fonction de l'évolution de leur état de santé physique et mentale, dont les intervenantes sont, par leur présence régulière auprès des personnes, les témoins privilégiés. La confiance qu'elles construisent au fil du temps, « patiemment et discrètement » (Molinier, 2005), avec les personnes auprès de qui elles interviennent, les place dans la situation la plus favorable ; et ce autant pour repérer précocement les signes d'une difficulté que pour ensuite trouver et mettre en œuvre les solutions les mieux appropriées pour y faire face au quotidien et assurer de cette façon l'objectif de bienêtre au cœur de leur métier.

Les formations à construire pour soutenir un tel métier doivent ainsi porter fondamentalement sur l'acquisition de connaissances approfondies sur les pathologies concernées et la diversité des cas, sur la psychologie des personnes, sur les techniques du «prendre soin ». En effet, on n'aide pas une personne âgée ou fragilisée et souvent désorientée à faire sa toilette, à se nourrir, à se déplacer, à s'occuper de son environnement..., comme on le ferait d'une personne dans la force de l'âge temporairement handicapée. Ces connaissances sont certes nécessaires quel que soit le lieu d'exercice du travail ; cependant, les modalités d'exercice du travail diffèrent selon qu'il est effectué à domicile ou en institution. Les responsabilités assumées sont de fait plus lourdes dans le cas du domicile, où les intervenantes agissent le plus souvent seules. Il importe donc aussi de former aux différentes pratiques qu'induisent ces modes d'intervention, à domicile ou en institution, dont on peut postuler un enrichissement mutuel : une plus grande technicité du travail à domicile versus une organisation des conditions de vie en maison de retraite sur un modèle moins hospitalier, plus respectueux des rythmes de chacun et plus attentionné à ses particularités ; toutes choses difficiles à réaliser dans le contexte actuel et dont souffrent aussi les personnels travaillant dans ces institutions (Dujarier, 2006) ${ }^{10}$.

10 « Les personnels de gériatrie ont le sentiment de "faire des vieux", de les rendre incontinents, abrutis, dépendants. Beaucoup disent avoir "honte" de la manière dont "on" les traite. Non pas tant à cause des actes de maltraitance sporadiques, mais plutôt parce que les personnes âgées que l'on accueille dans les hôpitaux ne sont plus en situation d'avoir une vie "humaine" (...) Ils sont contraints à l'inactivité, à l'inutilité et à la dépendance. Les actes de la vie quotidienne sont vidés de leur goût. » (Dujarier, 2006)
Si l'enjeu est important, en raison des besoins considérables en emplois rappelés en introduction, la question n'est pourtant pas nouvelle. La députée Paulette Guinchard-Kunstler l'avait déjà notée, en 1999, dans son rapport «Vieillir en France : enjeux et besoins d'une nouvelle orientation de la politique en direction des personnes âgées en perte d'autonomie », dans lequel elle précise : "Il apparaît clairement qu'autant au domicile qu'en hébergement, c'est autour de trois champs que s'accomplit le travail nécessaire à l'accompagnement des personnes âgées : les soins du corps, les actes de la vie quotidienne, la vie sociale et relationnelle. » Elle prônait ainsi : «La formation d'intervenants aux compétences élargies capables de répondre à l'ensemble des besoins des personnes en perte d'autonomie que ce soit au domicile privé de la personne ou en structure sanitaire ou médico-sociale. Outre l'intérêt évident que ce type de compétences peut présenter pour mieux adapter les réponses, ce nouveau profil professionnel favorisera le passage d'un exercice en hébergement vers un exercice à domicile et vice-versa. La perception des intervenants sera ainsi enrichie par une expérience diversifiée et la qualité de la prise en charge en sera d'autant accrue. »

Plus de dix ans plus tard, tout reste à faire. Les freins existants en 1999 se sont sans doute encore resserrés du fait de la loi Borloo de 2005 sur les services à la personne ; celle-ci ayant accentué la focalisation sur le domicile et la notion de service et, avec elle, celle de client et de prescripteur (Devetter et al., 2009, Chauvière, 2009). On constate aussi que les rapports sur l'emploi ou les formations, dans ce secteur, n'ont cessé de prôner l'indifférenciation des métiers ou des formations selon les publics (Le Bouler, 2006 ; Vérollet, 2007 ; Asseraf, 2010 ${ }^{11}$. Et pire, sans doute, parce qu'opérationnel dès la rentrée 2011, le choix a été fait de supprimer le BEP Carrières sanitaires et sociales pour créer un baccalauréat professionnel du même nom, avec deux options, conduisant à l'exercice de fonctions auprès de familles, d'enfants, de

\footnotetext{
${ }^{11}$ Nous faisons l'hypothèse que cette polyvalence des formations offertes est avant tout destinée à les rendre « attractives» pour les jeunes (filles), l'idée sous-jacente étant que, si les formations étaient spécialisées vers les adultes fragilisées, et en particulier vers les personnes âgées, elles ne seraient pas choisies par les jeunes filles dont on postule, inversement, qu'elles ont toutes le désir de s'occuper d'enfants.
} 
personnes âgées ou de personnes handicapées, dans le cadre de structures ou du domicile pour l'option « Services aux personnes », et d'établissements pour l'option «Accompagnant en établissements sanitaires, sociaux et médico-sociaux ». Ce Bac Pro est donc encore une fois polyvalent en termes de publics, quelle que soit l'option choisie. Le niveau du baccalauréat risque, par ailleurs, de poser la question de sa reconnaissance par la branche professionnelle de l'aide à domicile ; en effet, en tant que diplôme de niveau IV, il devrait être pris en compte selon un classement supérieur au DEAVS de niveau V. La question se pose aussi en établissement avec les diplômes d'aide-soignante ou d'aide médico-psychologique, également de niveau V. De plus, bien que prévu pour la rentrée 2011, il n'existe encore à ce jour aucun référentiel de formation permettant de savoir quels seront les enseignements délivrés et sous quelle forme. Le référentiel d'activités professionnelles laisse néanmoins supposer que les connaissances acquises seront très loin de couvrir l'éventail des situations rencontrées dans l'aide aux adultes fragilisés.

Ces orientations laissent ainsi perdurer une situation paradoxale : celle de clamer les besoins en personnels qualifiés pour faire face aux problèmes posés par le vieillissement de la population et par le souci de mieux prendre en compte les besoins des personnes handicapées ou des personnes atteintes de maladies graves, ... sans développer les formations susceptibles de conduire aux emplois répondant à ces besoins criants.
Les besoins croissants d'aide et d'accompagnement des personnes fragilisées par l'âge ou les évènements de la vie ne pourront être satisfaits sans changer profondément le cadre de l'organisation actuelle des formations. Il importe pour cela de considérer non pas le lieu d'exercice des activités (domicile ou institution) comme constituant le pivot des métiers et des formations mais l'objectif du travail, c'est-à-dire la construction d'une aide visant la préservation, le plus longtemps et le mieux possible, d'une autonomie inévitablement déclinante. Il est certes indéniable que cette aide est plus souvent requise à domicile, en raison du souhait de la plupart des personnes de vieillir chez elles et de politiques sociales qui visent à favoriser ce choix ; pour autant, ce n'est pas le domicile qui est au cœur du métier d'aide mais bien la personne bénéficiaire de l'aide.

Le vieillissement des populations mais aussi les progrès dans la prise en charge de maladies ou de handicaps graves rendent plus complexe la construction de ce travail d'aide, dans lequel la dimension des soins, au sens anglais du terme de care $^{12}$, a pris une importance accrue. Cela induit de concevoir des formations capables de soutenir les exigences de ce travail relevant, simultanément, de l'aide et du soin. Nos recherches auprès de salariées expérimentées de l'aide à domicile ont révélé que c'était d'abord et souvent exclusivement en mobilisant des ressources qu'elles avaient elles-mêmes construites au fil de leur pratique qu'elles avaient trouvé les réponses appropriées aux situations toujours particulières et "instables" des personnes aidées. L'expérience de ces " pionnières » et la richesse de leur réflexion sur leur métier représentent ainsi des atouts essentiels pour construire des référentiels de formation correspondant à la réalité des situations des personnes et de leurs besoins d'aide. 


\section{Bibliographie}

Asseraf G. (2009), Pour une "simplification de l'offre des certifications" dans le champ des services aux personnes fragiles, Rapport au Premier ministre.

Bonaïti C. (2008), « Le parcours des candidats à la validation par les acquis de l'expérience des titres et diplômes de niveau $\mathrm{V}$ : mieux vaut être accompagné et diplômé pour réussir ", Premières informations Premières synthèses, DARES, ${ }^{\circ} 34$.

Bressé S. (2004), « Les bénéficiaires des services d'aide aux personnes à domicile en 2000 », Études et Résultats, $\mathrm{n}^{\circ} 296$.

Chardon O., Estrade M. A. (2007), Les métiers en 2015, Centre d'analyse stratégique/DARES.

Chauviere M. (2009), « Qu'est-ce que la “chalandisation”? », Informations sociales, n ${ }^{\circ}$ 152, pp. 128-134.

DARES Analyses (2010), « Les métiers en 2009 », $\mathrm{n}^{\circ} 069$.

Debout C et Lo S-H. (2009), « L'allocation personnalisée d'autonomie et la prestation de compensation du handicap au 30 juin 2009 », Études et Résultats, $\mathrm{n}^{\circ} 710$.

Devetter F.X., Jany-Catrice F., Ribault T. (2009), Les services à la personne, Éditions La Découverte.

Doniol-Shaw G. (2005-a), La qualification des emplois de l'aide à domicile au regard de l'analyse de la validation des acquis de l'expérience dans le métier d'assistante de vie, Rapport LATTS, Marne-La-Vallée.

Doniol-Shaw G. (2005-b), « Les mises en situation reconstituées peuvent-elles être considérées comme des situations de travail ? Questions sur un dispositif de validation des acquis de l'expérience dans l'aide aux personnes à domicile ", Actes du $40^{e}$ Congrès SELF, Ergonomie et développement durable, Éditions de l'ANACT, pp. 238-245.
Doniol-Shaw G, Lada E, Dussuet A. (2007), Les parcours professionnels des femmes dans les métiers de l'aide à la personne. Leviers et freins à la qualification et à la promotion, Rapport LATTS, Marne-La-Vallée. http://www.travail-solidarite. gouv.fr/IMG/pdf/Latts_rapport_definitif_21_ mars_2008.pdf

Doniol-Shaw G, Lada E. (2009), « Le travail des aides à domicile auprès des personnes âgées en France : façons de dire, façons de faire » in Felix C., Tardif J., (éd.), Actes éducatifs et de soins, entre éthique et gouvernance, Actes du colloque international, Nice 4-5 juin 2009, Les ateliers, L'agir créatif: la prescription de l'action en question. Le cas de l'aide à domicile, mis en ligne le $1^{\text {er }}$ octobre 2010, URL : http://revel.unice.fr/symposia/actedusoin/index. html?id=643

Dujarier A.M. (2006), L'idéal au travail, Paris, PUF.

Dussuet A. (2005), Travaux de femmes - Enquêtes sur les services à domicile, Paris, L'Harmattan.

Fraisse G. (2009), Service ou servitude, essai sur les femmes toutes mains, Lormont, Le Bord de l'eau.

Guinchard-Kunstler P. (1999), Vieillir en France : enjeux et besoins d'une nouvelle orientation de la politique en direction des personnes âgées en perte d'autonomie. Rapport à M. le Premier ministre, La Documentation française.

Lada E. (2010), « Le travail d'aide à domicile et les politiques de marchandisation font-ils bon ménage ? Le travail des salariées de l'aide à domicile à l'épreuve des transformations des régimes de care en France "), Colloque international La dimension relationnelle des métiers de service : Cache-sexe ou révélateur du genre, Lausanne, 2-3 septembre 2010. http://www.unil.ch/webdav/site/liege/shared/ TexteEmmanuelleLada.pdf 
Laugier S., Molinier P., Paperman P. (coord) (2009), Qu'est-ce que le care? Souci des autres, sensibilité, responsabilité, Paris, Payot.

Le Bouler S. (2006), Personnes âgées dépendantes : bâtir le scénario du libre choix, Second rapport de la mission « Prospective des équipements et services pour les personnes âgées dépendantes », Centre d'analyse stratégique.

Marquier R. (2010), « Les intervenantes au domicile des personnes fragilisées en 2008 », Études et Résultats, $\mathrm{n}^{\circ} 728$.

Marquier R. (2008), « Préparer le diplôme d'État d'auxiliaire de vie sociale par la validation des acquis de l'expérience », Études et Résultats, n 658.

Molinier P. (2005), « Le care à l'épreuve du travail. Vulnérabilités croisées et savoir-faire discrets », in Paperman P., Laugier S. (coord). Le souci des autres.
Éthique et politique du care, Paris, Éditions de l'EHESS, pp. 299-316.

Pattaroni L. (2005), « Le care est-il institutionnalisable ? ", in Paperman P., Laugier S. (coord). Le souci des autres. Éthique et politique du care, Paris, Éditions de 1'EHESS, pp. 177-200.

Presse M. C. (2004), « Entre intention et réalité, les obstacles à la validation des acquis », Éducation permanente, $\mathrm{n}^{\circ} 158$, pp. 141-151.

Vasselin K. (2002), « Faire le ménage : De la condition domestique à la revendication d'une professionnalité », Piotet F, La révolution des métiers, PUF, pp. 77-98.

Verollet Y. (rapporteur) (2007), Le développement des services à la personne, Rapport du Conseil économique et social.

\section{Résumé}

\section{Quels diplômes pour le secteur de l'aide à la personne? \\ Ghislaine Doniol-Shaw}

En dépit de besoins élevés et croissants dans le secteur de l'aide aux personnes, et avant tout aux personnes âgées, il n'existe, en France, aucune filière de formation spécifique. Cet article, qui s'appuie sur des recherches sur l'aide aux personnes à domicile, interroge ce paradoxe. Ainsi, la polyvalence des formations, orientées à la fois vers les tout-petits et les personnes âgées, est en contradiction avec la réalité du travail offert, qui ne concerne quasiment que les personnes âgées. Dès lors, elle constitue un frein à la qualification et à la reconnaissance de l'expérience professionnelle des salariées. De nouvelles voies de formation, qui rapprochent l'aide et le soin et questionnent les frontières tracées entre le sanitaire et le social, sont proposées et discutées.

Mots clés

Service aux particuliers, aide à domicile, formation initiale, diplôme, validation des acquis Journal of Economic Literature : L 84, J 24 